\title{
CRESCIMENTO CRANIANO NA CRIANÇA
}

\author{
M. LUIZA CONSTANTE ROSADO* - MARCO A. BARBIERI** - HELOISA BETTIOL** \\ UILHO A. GOMES ***; - M. VALERIANA L. MOURA-RIBEIRO *
}

\begin{abstract}
RESUMO - Os autores fazem ampla revisăo dos estudos e pesquisas realizadas sobre crânio em recém-nascidos e lactentes, procurando analisar e correlacionar os dados antropométricos com os váríos fatores biológicos e sociais maternos.
\end{abstract}

Skull growth in children.

SUMMARY - The authors review papers and researches about skull growth in newborns and infants; they analysed and correlated anthropometric data with maternal biological and social factors.

As curvas de crescimento fetal permitem não somente classificar os recém-nascidos (RN) de acordo com os parâmetros de crescimento intra-uterino, como fornecem meios para acompanhar e avaliar o desenvolvimento dos nascidos pré-termo e/ou desnutridos intra-útero. Os valores das medidas da circunferência craniana obtidos evolutivamente nas crianças fornecem, de modo indireto, a estimativa do crescimento encefálico durante os primeiros anos de vida. Entretanto, o tamanho da cabeça pode ser fator meramente familiar ou reflexo do tamanho da criança 64 . Há duas décadas, aproximadamente, Dobbing iniciou estudos em mamiferos sobre o crescimento encefálico e verificou que este não se processava de modo linear, simétrico 16-21.

$\mathrm{Na}$ espécie humana, o período de desenvolvimento encefálico mais acelerado, da $12^{\text {* }}$ até a $18^{*}$ semana gestacional, está associado particularmente à multiplicação neuronal. Outro surto de desenvolvimento ocorre da $28^{a}$ semana gestacional até em torno do terceiro ano de vida pós-natal. Na primeira metade deste, inicia-se multiplicação glial que perdura até o $24^{\circ}$ dia de vida pós-natal. Já na segunda metade, começa a ocorrer mielinização rápida, dependente em suas bases da integridade funcional das células gliais 65,66 . Portanto, quando 0 grande surto de crescimento encefálico se processa, a maior parte dos neurônios já está formada, com exceção dos microneurônios cerebelares e de parte de neurônios do tronco cerebral. Dessa forma, a interferência de fatores mórbidos restritivos, nesta fase, não deve provocar alteraçōes significativas no total de neurônios. Todavia, poderá haver comprometimento glial, da mielinização, da arborização dentrítica e das interconexões sinápticas 51,59. O surto normal do crescimento cerebelar pode ser constatado a partir da $30^{4}$ semana intrauterina, cessando no final do primeiro ano de vida. As influências restritivas podem provocar alteração numérica dos microneurônios granulares do cerebelo, sem redução do número das células de Purkinje 16,66,67. Os animais com cerebelo assim afetado são desajeitados, com inépcia motora, alteração essa reconhecida também na espécie humana 10. Alguns estudos histopatológicos têm assinalado ausência de lesões encefálicas focais a partir de distúrbios nutricionais, outros comprovam distorções na relação quantitativa, bem como alterações neuroquímicas 50,66,67. Experimentalmente, em ratos, constatou-se redução em $37 \%$ de interconexões sinápticas para cada neurônio, após período de desnutrição moderada 21 .

Faculdade de Medicina de Ribeirão Preto, Universidade de São Paulo (USP): * Departamento de Neuropsiquiatria e Psicologia Médica; * Departamento de Puericultura e Pediatria; \&** Departamento de Medicina Social. 
A hipótese de que o tamanho da cabeça equivale ao volume dos vários componentes intracranianos tem sido comprovada, tanto de maneira direta 26,41 como indireta, por correlação perimetro craniano (PC) e estudo radiológico do crânio 7 , correlação $\mathrm{PC}$ e coeficiente intelectual $2,27,30,53,59, \mathrm{PC}$ e número de células encefálicas 40,54 . A maioria dos estudos que relaciona coeficiente intelectual com circunferência crânica, tem sido realizada em crianças de peso inferior a $2500 \mathrm{~g}$. A probabilidade da criança de baixo peso sobreviver, no transcorrer do primeiro ano de vida, é inferior à daquelas nascidas com peso dentro das variáveis normais 38,40 . Isto porque, além da baixa resistência às infeç̧ōes e à menor capacidade de produção de imunidade celular, $o$ RN não raramente pode apresentar anomalias anatômicas 63 e maior exposição à desnutrição pré- e pós-natal por pertencer a classes sócio-econômicas menos favorecidas 40,51. Por outro lado, as crianças com valores de PC acima de dois desvios padrões da média para a idade, de forma geral, devem ser submetidas a estudos complementares para verificação de afecçăo neurológica. Assim sendo, ao avaliar um dado paciente, devem ser analisadas as medidas e proporcionalidade ou não existentes entre tamanho da cabeça e altura do indivíduo.

A possivel associação entre $\mathrm{PC}$ pequeno e/ou mau desempenho intelectual com baixo nivel sócio-econômico-cultural é discutida por vários autores $4,22,23,56$, como entre o mau desempenho intelectual e prematuridade 24 e entre prematuridade e baixo peso associado a classes sociais menos favorecidas, também motivos de discussão 4 . Alguns autores apontam ao PC das crianças da zona urbana, superior aos da rural 24,43,59, implicaçóes de caráter nutricional. Jansen 34 , entretanto, afirma que meninos das classes sócio-econômicas privilegiadas rurais tếm PC major em todas as faixas etárias. Fitzhardinge e Steven 27 estudaram $93 \mathrm{RN}$ de baixo peso para idade gestacional, sem comprometimento neurológico ao nascimento e com $\mathrm{PC}$ adequado à idade gestacional; no acompanhamento prospectivo dessas crianças do ponto de vista clínicoantropométrico e psicológico até a adolescência, pode ser constatado, em $50 \%$ dos meninos e $36 \%$ das meninas, mau desempenho escolar. Babson e Henderson 1 demonstraram diminuição dos valores do $\mathrm{Ql}$ em crianças de baixo peso cujo $\mathrm{PC}$ com um ano de vida estava abaixo do décimo percentil, em comparaçăo àquelas cujo $\mathrm{PC}$, na mesma idade, estava entre percentil $25^{\circ}$ e $75^{\circ}$; entretanto, aos 10 anos de idade, não mais foram evidenciadas as diferenças de QI, embora se mantivesse a diferença no PC, sugerindo que o retardo no crescimento intra-uterino, desacompanhado de anormalidades neurológicas, não interfere no desenvolvimento físico e mental de crianças mantidas em ambiente estimulador.

As medidas da circunferência crânica de $\mathrm{RN}$ e lactentes são correlacionadas a fatores maternos, placentários ou fetais, sendo unânime a constatação da relação direta entre PC e sexo em todas as idades, isto é, o PC é maior cerca de $1 \mathrm{~cm}$ nos meninos $6,36,37,42,43,52,53,55,60$. Existe, também, correlação positiva do $P C$ com a idade gestacional, o peso e o comprimento do RN 11. Miller e Hassanein 48, procurando determinar padrões de impedimento de crescimento fetal, utilizaram a relação $\mathrm{PC} / \mathrm{com}$ primento baseados em estudo de Scammon e Calkins (1929) segundo o qual para cada $10 \mathrm{~mm}$ de aumento no comprimento do feto havia aumento de $6,5 \mathrm{~mm}$ na circunferência occipito-frontal; analisaram a relação $\mathrm{PC} /$ comprimento em $1437 \mathrm{RN}$ com idade gestacional variando de 30 a 44 semanas, procurando verificar a proporção ou não com o crescimento esquelético; as médias encontradas para crianças brancas e negras, meninos e meninas, de mães nulíparas ou multíparas e em todas as idades gestacionais foram semelhantes, estatisticamente. Os meninos brancos e negros combinados, apresentaram média de $\mathrm{PC}$ maior do que as meninas brancas e negras combinadas, em cada intervalo de idade gestacional considerada a partir da $37^{4}$ semana 32,52 . Nas crianças pré-termo, de peso adequado para a idade gestacional, o crescimento da cabeça se faz de maneira acelerada nos primeiros meses de vida pós-natal 12,29,39,33,44,57, bem como nas de baixo peso para a idade gestacional 46. Nestas últimas os valores de PC permanecem menores até o final do primeiro ano de vida 1 . Blidner et al.6, em estudo controlado de $1231 \mathrm{RN}$, observaram que os meninos tendem a ser mais pesados, mais compridos, de cabeça e tórax maiores que as meninas. Existe forte correlação do PC e medidas corporais com a idade gestacional $2,12,42,62,56,57,60$. A idade gestacional constitui dado pouco preciso 6,61 , devendo ser completadas as informaçóes fornecidas pela mãe com a avaliação da maturidade neurológica do RN pelo método de Dubowitz.. Tem sido constadado também retardo no crescimento intra-uterino, associado a anomalias placentárias e nutrição materna $8,9,48,61$. Davies 13 , entretanto, afirma que a circunferência crânica está mais relacionada às açōes ambientais intra-uterinas que o peso e comprimento; é ainda menos sensível a influências maternas, tais como 
diabetes, paridade alta, número ou frequência de aleitamento, desnutrição, hipertensăo, ou a anomalias placentárias.

Davies 14 chama a atenção para a recuperação do crescimento em RN hipotrófico sem retardo prolongado no crescimento intra-uterino e sem genótipo próprio de comprimento maior. Naeye et al.51 demonstraram que há diminuição real do peso dos órgăos de fetos de mães desnutridas e com baixo ganho ponderal durante os dois últimos trimestres de gestação; nos fetos de até 33 semanas foi verificada diminuição do número de células nos vários órgãos; todavia, naqueles com 37 ou mais semanas havia diminuição do conteúdo citoplasmático das células. Observaram, ainda, que: o comprimento do RN e seu encéfalo se mantiveram poupados; as mulheres previamente bem nutridas getaram crianças cada vez maiores, enquanto as permanentemente subnutridas, crianças ou fetos cada vez menores. Na Holanda, em 1944, durante a guerra, para mulheres grávidas que tiveram suas dietas drasticamente diminuídas no último trimestre da gestação, constatou-se redução de aproximadamente $10 \%$ no peso médio dos bebês 59 .

O maior tamanho do cérebro de crianças nascidas de māes bem nutridas faz pensar na possibilidade de que o crescimento encefálico atinga a plenitude no potencial genético sob condições ótimas de nutrição 49,59 . Levando-se em consideração os estudos que associam malnutrição nos primeiros anos de vida com redução do peso encefálico 65,66 e diminuição do número de células nervosas com deficiências neuropsico-

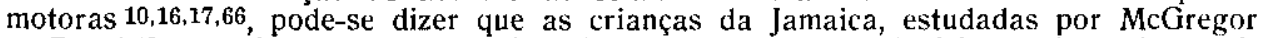
\& Desai 43, constituem grupo de risco importante de não adquirirem pleno desenvolvimento neuromotor e/ou intelectual. Em relação à influência da classe social sobre o tamanho da cabeça do recém-nascido, Barbieri 3 enfatiza que: 'o que se pretende é tentar articular o biológico em um contexto social, na fase em que as causas sociais podem desencadear e transformar os fatores biológicos. Assim, por exemplo, a maior mortalidade infantil ou maior proporção de baixo peso nos filhos de mulheres nas idades extremas e o maior risco nos recém-nascidos de grandes multíparas têm uma explicação biológica. Mas, por sua vez, são os determinantes sociais do comportamento reprodutivo (fecundidade), condicionando que as mulheres dos grupos sócioeconômicos baixos tenham uma reprodução de início precoce, elevada e prolongada, com maior proporção de nascimentos nos grupos de maior risco 34'. O tabagismo durante a gravidez e a idade materna à época do parto, em nosso meio ${ }^{3}$, se mantêm fortemente influenciados pela classe social materna 3,31. Alguns autores também estudaram, como fator marcadamente social, o trabalho materno fora do lar $3,4,60$ e, ainda, a estatura da mãe $4,5,23,51$.

Woiski et al.68, em estudo realizado no Hospital das Clínicas da Faculdade de Medicina de Riberão' Preto (FMRP) elaboraram tabelas classificando 524 RN consecutivos normais a termo, segundo sexo, peso, estatura, perímetro torácico e cefálico; foi encontrada a média de $P C$ de $35 \mathrm{~cm}$ para meninos e $34 \mathrm{~cm}$ para meninas; chamam atençāo para os valores médios, inferiores em relação à literatura; ressaltam, também, a relação constante entre comprimento e os perimetros craniano e torácico, como atuxiliar na avaliação das proporçōes corporais em crianças. Marcondes et al.45 em 1971 realizaram amplo estudo antropométrico em 9258 crianças brasileiras de 0 a 12 anos, encontrando valores médios de $\mathrm{PC}$ de $34,26 \mathrm{~cm}$ em 100 meninas RN e $35,01 \mathrm{~cm}$ em 134 meninos, resultados esses inferiores aos estudos europeus e norte-americanos, todavia semelhantes aos de Diament 15. Diament 15 , estudando 113 crianças de 0 a 12 meses, elaborou tabelas de médias e desvios padróes de $\mathrm{PC}$ segundo sexo e grupo etário, apontando para os $\mathrm{RN}$ do sexo masculino a média de $34,61 \mathrm{~cm}$ e, para os do sexo feminino, $34,05 \mathrm{~cm}$. Martins-Filho 47 (em Campinas) e Joaquim 35 (em Brasília) publicaram os dados relativos a peso, estatura e perimetro cefálico em crianças normais, obtidos intra-útero. Barbieri 4, em estudo longitudinal de crianças de 0 a 2 anos nascidas de baixo peso no Hospital das Clinicas da FMRP, realizou medidas seriadas de peso, comprimento, PC, PT e perimetro braquial desde 48 horas de vida até 2 anos e elaborou curvas de crescimento, bem como estudou as relações peso/ estatura, comprimento tronco-cefálico/estatura e PC/PT e as variedades de crescimento de peso, estatura e PC do nascimento até dois anos de idade. Siqueira et al.58, em estudo retrospectivo, elaboraram tabelas de valores médios e desvio-padrão de peso, estatura e perímetro cefálico de 382 crianças de classe sócio-econômica privilegiada e compararam os resultados aos de estudos semelhantes; as crianças brasileiras de classes sociais mais favorecidas tèm crescimento físico similar ao das crianças de paises desenvolvidos, reforçando a idéia de ocorrer influência de fatores sociais no desenvolvimento físico do indivíduo. 
Dessa forma, o conhecimento amplo dos fatores que influenciam o desenvolvimento cerebral da criança deve ser atualizado e constantemente revisado, procurando não só estabelecer comparações entre as diferentes populações como, também, detectar fatores que podem comprometer em maior ou menor intensidade o neurodesenvolvimento e a potencialidade intelectual.

\section{REFERENCIAS}

1. Babson SG, Henderson NB - Fetal undergrowth: relation of head growth to later intellectual performance. Pediatrics 53:890, 1974.

2. Babson SG, Renda GF - Growth graphs for the elinical assessment of infants of varying gestational age. J Pediatr 69:814, 1976.

3. Barbieri MA - Saúde materno-infantil e classe social: alyuns aspectos do periodo perinatal da mâe e do recém-nascido. Tese (Livre-Docência). Faculdade de Medicina de Ribeirão Preto, Universidade de Săo Paulo. Ribeirão Preto, 1985.

4. Barbieri MA - Estudo longitudinal do desenvolvimento físico de crianças de zero a dois anos de vida nascidas com baixo peso no Hospital das Clinicas da Faculdade de Medicina de Ribeirão Preto da Universidade de São Paulo. Tese (Doutorado). Faculdade de Medicina de Ribeirão Preto, Universidade de Sāo Paulo. Ribeirão Preto, 1975.

5. Bjerde I, Varendh $G-A$ study of biological and economic factors in low birth-weight. Acta Paediatr Scand 64:605, 1975.

6. Blidner IN, McClemont S. Anderson GD, Sinclair JC - Size-at-birth standards for an urban Canadian population. Can Med Ass J 130:133, 1984.

7. Bray PF, Shields WD, Wolcott GJ - Occipito-frontal head circumference: an accurate measure of intracranial volume. J Pediatr 75:303, 1969.

8. Butler NR, Alberman ED - Perinatal problems: the second report of the 1958 British Perinatal Mortullty Survey. Livingstone, Edinburgh, 1969.

9. Chase HP, Welch NN, Dabiere CS - Alterations in human brain biochemistry following intrauterine growth retardation. Pediatrics 50:403, 1972.

10. Craviotto J - Desnutriçāo grave $A$ desenvolvimento das aptidões motoras na criança. Anais Nestlé $107: 21,1982$.

11. Dangerfield RH, Taylor CJ - Anthropometric standards for term neonates. Early Human Develop 8:225, 1983.

12. Davies PA, Davies JP - Very low birth-weight and subsequent head growth. Lancet $2: 1216,1970$.

13. Davies DP - Size of bith and growth in the first year of life of babies who are over weight and under weight at birth. Proc Nutr Soc 39:25, 1980.

14. Davies DP - O recém-nascido hipotrófico. Anais Nestlé 40:3, 1984.

15. Diament AJ - Contribuição para a sistematizaçău do exame neurológico de crianças normais no $1^{\circ}$ ano de vida. Tese (Doutorado) Faculdade de Medicina, Universidade de São Paulo. São Paulo, 1967.

16. Dobbing J, Hopewell JW, Lynch A - Vulnerability of developing: VII. permanent deficit of neurons in cerebral and cerebellar cortex following early mild undernutrition. Exp Neurol 32:439, 1971.

17. Dobbing J, Sands J - Vulnerability of developing brain. Biol Neonate 19:363, 1971.

18. Dobbing $J$, Sands $J-$ Quantitative growth and development of human brain. Arch Dis Childh 48:757, 1973.

19. Dobbing $J$, Smart JL - Vutnerability of dereloping brain and behaviour. $\mathrm{Br}$ Med Bull $30: 164,1974$.

20. Dobbing $\mathbf{J}$ - The later growth of the brain and its vulnerability. Pediatrics 53:2, 1974.

21. Dobbing $\mathbf{J}-$ Carência nutricional, retardamento do crescimento e sistema nervoso. Anals Nestle 107:5, 1982.

22. Drillien CM, Richmond F - Prematury in Edlnburgh. Arch Dls Childh 31:390, 1956. 
23. Drillien CM - The growth and development of the prematurely born infant. Livingstone, Edinburgh, 1964, pg 56.

24. Engsner G - Brain growth in privileged and non privileged Ethiopian children. Environ Child Health 19:357, 1973.

25. Eng'sner G, Habte D, Sjogren $I$ - Brain growth in children with Kwarshiorkor. Acta Paediat Scand 63:687, 1974.

26. Epstein HT, Epstein EB - The relationship between brain weight and head circumference from birth to age 18 years. Am J Phys Anthrop 48:471, 1978.

27. Fitzhardinge PM, Steven EM - The small-for-date infant: I. Later growth patterns. Pediatrics $49: 671,1972$.

28. Fujimura M, Seryu $J$ - Velocity of head growth during the perinatal period. Arch Dis Childh 52:105, 1977.

29. Fujimura $M$, Seryu $J$ - Factors which influence the timing of maximum growth rate of the head in low-birth-weight infants. Arch Dis Childh 52:113, 1977.

30. Gross SJ, Kosmetatos N, Grimes CT, Williams ML - Newborn head size and neurological status. Am J Dis Childh 32:753, 1978.

31. Hardy JB, Mellitis ED - Does material smoking during pregnancy have a long-term effect on the child? Lancet $2: 1332$, 1972 .

32. Hassanein $\mathbf{K}$, Miller HC - Diagnosis of impaired fetal growth in newborn infants. Pediatrics 48:5̌11, 1971.

33. Illingworth RS, Lutz W - Head circumference of infants related to body weight. Arch Dis Childh 40:672, 1965.

34. Jansen $\mathbf{J}-$ Head circumferenece in Danish children: allometric growth. Dan Med Bull 29 (Suppl 1): 1, 1982.

35. Joaquim MCM, Brandt JDC, Jacomo AJD, Lisboa AMJ - Crescimento intra-uterino. Bol of Sanit Panam 85:137, 1978.

36. Kantero RL, Tiisala $R$ - Growth of head circumference from birth to 10 years. Acta Paediatr Scand 220 (Suppl 1): 27, 1971.

37. Karlberg P, Engstrom I, Lichtenstein H, Klackenterg G, Klackenberg-Larsson I, Stensson J, Svennberg I - The development of children in a Swedish urban community: a prospective longitudinal study. Acta Paediatr Scand 187 (Suppl 1): 48, 1968.

38. Landicho B, Lechtig A, Klein RE - Anthropometric indicators of low birth weight. J Trop Ped 31:301, 1985.

39. Largo $\mathrm{RH}$, Duc $\mathrm{G}$ - Head growth and changes in head configuration in healthy preterm and term infants during the first six months of life. Helv Paediatr Acta 32:431, 1977.

40. Lechtig A - Causas de bajo peso al nascer en latinoamerica. Arch Latinoam Nutr 27 (Suppl 1): 147, 1977.

41. Lemmons JA, Schreiner RL, Gresham EL - Relationship of brain weight to head circumference in early infancy. Hum Biol 53:351, 1981.

42. Lubchenco LO, Hansman $\mathrm{C}$, Boyd $\mathbf{E}-$ Intrauterine growth in lenght and head circumference as estimated from live births at gestational ages from 26 to 42 weeks. Pediatrics $37: 403,1966$.

43. McGregor SMG, Desai C - Head circumference of Jamaican infants. Develop Med Child Neurol 15:441, 1973.

14. Mamelle $N$, Lazar $P$ - Étude allométrique de la croissance relative de la tête et du corps chez le foetus et le nouveauné. J Gyn Obst Blol Repr 8:703, 1979.

45. Marcondes E, Berquó ES, Yunes J, Luongo J, Martins JS, Zacchi MAS, Levy MSF, Hegg $\mathbf{R}-$ Estudo antropométrico de crianças brasileiras de 0 a 12 anos. Anais Nestlé $84: 146,1971$.

46. Marks KH, Maisels MJ, Moore E, Gifford $K$, Friedman $Z$ - Head growth in sick premature infants: a longitudinal study. J Pediatr 94:282, 1979.

47. Martins-Filho J - Contribuiçāo ao estudo do crescimento intra-uterino. Tese (Doutorado). Faculdade de Ciências Médicas, Universidade Estadual de Campinas. Campinas, 1972. 
48. Miller HC, Hassanein $\mathrm{K}$ - Fetal malnutrition in white newborn infants: maternal factors. Pediatrics 52:504, 1973.

49. Miller RW, Blot WJ - Small head size after 'in utero' exposure to atomic radiation. Lancet $2: 784,1972$.

50. Minkowski A, Larroche JC, Vignald J, Dreyfus-Brisac C, Saint-Anne Dargassies $\mathbf{S}-$ Development of the nervous system in early life. In Falkner $F$ (ed): Human Development. Saunders, Philadelphia, 1966. Apud Drillien CM - The small-for-date infant: etiology and prognosis. Ped Clin North Am 17:9, 1970.

51. Naeye RL - Effects of maternal nutrition on the human fetus. Pediatrics 52:494, 1973.

52. Nellhaus G - Head circumference from birth to eighteen years. Pediatrics 41:106, 1968.

53. Nelson KS, Deutshberger J - Head size at one year as a predictor of four-year IQ. Develop Med Child Neurol 12:487, 1970.

54. O'Connell EJ, Feldt RH, Stickler G - Head circumference, mental retardation and growth failure. Pediatrics $36: 62,1965$.

55. Palti H, Adler B - Anthropometric measurements of the newborn: sex differences and correlations between measurements. Hum Biol 47:523, 1975.

56. Reinhardt M, Gautier R, Reinhardt NM - A study of 204 consecutive deliveries in Abidjan anthropometric data of newborn, mothers and placentas. Helv Paediatr Acta 33:24, 1978.

57. Sher PK, Brown SB - A longitudinal gtudy of head growth in pre-terms infants: I. Normal rates of head growth. Develop Med Child Neurol 17:705, 1975.

58. Siqueira AAF, Andrade $\mathrm{J}$, Almeida PAM - Peso, comprimento e perímetro cefálico do criancas brasileiras de classe social elevada. Rev Paul Med 97:58, 1981.

59. Stein $Z$, Susser $M$, Saenger G, Marolla $F-$ Famine and Human Development: The Dutch Hunger Winter of 1944-1945. Oxford Univ Press, New York, 1975.

60. Ulrich $\mathbf{M}$ - Fetal growth patterns in a population of Danish newborn infants. Acta Paediatr Scand 292 (Suppl 45): 5, 1982.

61. Usher R, McLean F - Intrauterine growth of live born caucasian infants at sea level: standards obtained from measurements in 7 dimensions of infants born between 25 and 44 weeks of gestation. J Pediatr 74:901, 1969.

62. Waaler PE - Anthropometric studies in Norwegian children. Acta Paediatr Scand 308 (Supp1 1): 3, 1983.

63. Warkany J, Monroe VB, Sutherland BS - Intrauterine growth retardation. Am J Dis Childh 102:249, 1961.

64. Weaver DD, Christian JC - Familial variation of head size and adjustment for parental head circumference. J Pediatr 96:990, 1980.

65. Winick M - Malnutrition and brain development. J Pediatr 74:667, 1969.

66. Winick M, Rosso $\mathrm{F}$ - The effect of severe early malnutrition on cellular growth of human brain. Pediat Res 3:181, 1969.

67. Winick M - Cellular growth in intrauterine mainutrition. Ped Clin N Am 17:69, 1970.

68. Woiski JR, Romera J, Raya LC, Correa CEC, Lima EC Fitho - indices somáticos dos recém-nascidos normais no Hospital das Clínicas de Ribeirão Preto. Rev Paul Med $66: 12,1965$. 